\title{
Accounting for Proof Test Data in a Reliability Based Design Optimization Framework
}

\author{
Gerhard Venter (gventer@sun.ac.za)* \\ University of Stellenbosch, Stellenbosch, South Africa \\ Stephen J. Scotti (Stephen.J.Scotti@nasa.gov) ${ }^{\dagger}$ \\ NASA Langley Research Center, Hampton, VA 23681-2199
}

This paper investigates the use of proof (or acceptance) test data during the reliability based design optimization of structural components. It is assumed that every component will be proof tested and that the component will only enter into service if it passes the proof test. The goal is to reduce the component weight, while maintaining high reliability, by exploiting the proof test results during the design process. The proposed procedure results in the simultaneous design of the structural component and the proof test itself and provides the designer with direct control over the probability of failing the proof test. The procedure is illustrated using two analytical example problems and the results indicate that significant weight savings are possible when exploiting the proof test results during the design process.

\section{Nomenclature}

$\boldsymbol{R} \quad$ Correlation matrix

$\mu \quad$ Mean value

$\bar{G} \quad$ Linearized limit state function

$\sigma \quad$ Stress value

$\tilde{\sigma} \quad$ Allowable stress

*Professor, Department of Mechanical and Mechatronic Engineering, Stellenbosch, South Africa, AIAA Associate Fellow

${ }^{\dagger}$ Chief Engineer for Structures and Materials, Research Directorate, NASA Langley Research Center, AIAA Member 
$\tilde{L} \quad$ Allowable service load

A Proof test success event

$b \quad$ Width

F $\quad$ Failure event

$f \quad$ Objective function

$G \quad$ Limit state function

h Height

L $\quad$ Service load

$l$ Length

$M \quad$ Number of analysis in a Monte Carlo simulation

$m \quad$ Number of proof loads

$m f \quad$ Number of failure modes

$m p \quad$ Number of proof failure modes

$N \quad$ Normal distribution

$n \quad$ Number of design variables

$P \quad$ Probability

$p \quad$ Proof load magnitude

$P_{f} \quad$ Probability of failure estimated by a Monte Carlo simulation

$q \quad$ Distributed load

$s \quad$ Standard deviation

SF Safety factor

$x \quad$ Design variable

\section{Introduction}

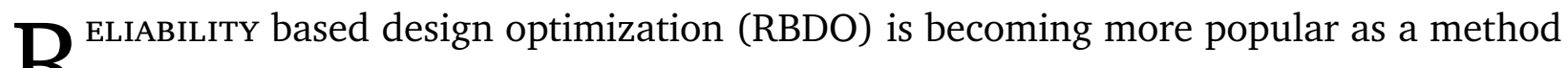
Rfor designing reliable structures when presented with uncertainty in the problem parameters. There are currently a large number of research projects that investigate the efficient application of RBDO to real life scenarios. These include methods for efficiently estimating the probability of failure (e.g., Grandhi and Wang ${ }^{1}$ ) as well as methods for updating the uncertainty as more data (typically as a result of additional testing) becomes available (e.g., Acar et al. ${ }^{2}$ ). In general these studies present numerically efficient methodologies that provide the most benefit (typically in the form of weight savings) by using RBDO.

This paper investigates an alternative RBDO approach that may enable significant weight savings, without accepting higher risk. The approach is best explained by considering the life cycle of a structure as outlined by the USAF Aircraft Structural Integrity Pro- 
gram (ASIP). The ASIP considers the full life cycle of a vehicle consisting of: requirement specification (Task 1); design and development (Task 2); verification testing (Task 3); test evaluation/analysis for certification and sustainment (Task 4); and force management/sustainment (Task 5). The ASIP is focused on designs that result in robust vehicle operations, and it is usually acceptable to incur performance (i.e., weight) penalties that minimize periodic inspections and/or repairs. The ASIP process only provides loose coupling between the outlined tasks. The present paper will propose an integrated engineering approach that combines several of these tasks to enable significant performance enhancements by utilizing proof (or acceptance) test data during the RBDO of structural components. A key assumption in the present work is that every component that is manufactured is proof tested and will only enter into service if it passes the proof test. The research was motivated by the current design process at NASA, which can be summarized as:

1. Mostly a deterministic design process

2. Typically only a relatively small number of components are manufactured

3. Virtually all components are proof tested before entering service

The advantage of considering a RBDO approach over the traditional deterministic approach within the NASA context has already been illustrated (e.g., Mason and Krishnamurthy $^{3}$ ). The goal here is to show how the RBDO process can further benefit by also exploiting proof test data in cases where it is available. In this research, a proof test is considered as a test that simulates the service load and that is performed on every component before entering service. Currently, proof tests are conducted at NASA, but the results of the proof tests are not directly included in the design process. The influence of proof testing on the strength distribution has been previously reported by Herbert and Trilling ${ }^{4}$ in the context of thermal loading. Herbert and Trilling ${ }^{4}$ accounted for the reduced uncertainty in the strength distribution in their RBDO approach, but did not include the proof test as part of the design process. In contrast Acar et al. ${ }^{2}$ considered the influence of future uncertainty reduction measures in the form of structural tests on aircraft safety during the design process, resulting in simultaneous design of the structure and the tests. However, the work by Acar et al. ${ }^{2}$ concentrated on future tests that are aimed at the reduction of uncertainty (for example coupon tests) and not proof tests. In addition, the additional information is used to update the uncertainty in the mean failure stress using a Bayesian update procedure. In the current work proof tests will be considered and no Bayesian updating will be performed.

The proposed RBDO approach aims to provide significant weight reductions, while maintaining high levels of reliability. Also, the methodology provides the designer with 
direct control over the probability of failing the proof test itself. Controlling the probability of failing the proof test is a desirable and extremely important feature. In some cases, where the cost of failing the proof test is bearable, larger weight savings can be realized by accepting higher risk. In other cases, where a proof test failure would be extremely costly, the risk of failing the proof test can be reduced but will increase the component weight.

Although the current work was inspired by the NASA design process where virtually all components are proof tested before entering service, the methodology could also be used to investigate the addition of simple proof tests to components that are currently not proof tested. For example, commercial aircraft are typically type certified. Type certification involves extensive testing that is performed on a small number of prototypes, while the production planes that are delivered to the customers are only subjected to limited testing. In these cases, it could be worthwhile to investigate the cost benefit of adding simple proof tests to each of the production aircraft as well. An example may be to connect actuators to the wings to perform a simple bending proof test on each aircraft.

\section{Reliability Based Design Optimization}

The traditional deterministic design process accounts for uncertainty in the problem parameters by using safety factors. Safety factors for airframe structural design have a long heritage that has evolved over many years. The safety factor approach provides for structural reliability by requiring that the design structural strength be greater than the stresses induced by external loads by at least the factor of safety. Historically, the safety factor accounts for uncertainties such as the occurrence of extreme loads, inaccuracies of stress prediction methods, variability of materials, variability in fabrication workmanship, and structural strength deterioration over the lifetime of an airframe. The values used for safety factors have evolved over many years and typically have been reduced as greater knowledge and reduced variability in materials and processes were obtained. ${ }^{5}$

The traditional deterministic design process is illustrated by the following example, where a structural component is designed subject to a stress constraint as follows:

$$
\begin{aligned}
\text { Minimize: } & f(\boldsymbol{x}) \\
\text { Such That: } & \sigma_{\max }(\boldsymbol{x}) \leq \sigma_{\text {fail }} \\
& x_{i}^{\text {lower }} \leq x_{i} \leq x_{i}^{\text {upper }} \quad i=1, n
\end{aligned}
$$

In Eq. $1, f(\boldsymbol{x})$ represents the objective function (typically weight), $\boldsymbol{x}$ the vector of $n$ design variables, $\sigma_{\max }$ the maximum stress in the component and $\sigma_{\text {fail }}$ the failure stress. Upper

and lower bounds on the design variables are specified by $x_{i}^{\text {lower }}$ and $x_{i}^{\text {upper }}$ respectively. 
To deal with uncertainty, a safety factor is typically used to obtain a design load $\tilde{L}$ for calculating the maximum stress $\sigma_{\max }$. In addition, the stress limit is adjusted to obtain an allowable stress limit $\tilde{\sigma}_{\text {fail }}$. A standard approach for generating an allowable stress limit is to obtain data from simple coupon or element tests and then perform a statistical analysis to estimate an allowable stress which has a specified, low probability of causing a structural failure. The allowable stress limit is calculated to be the mean failure stress from the tests, reduced by the product of the test standard deviation times a stress limit adjustment factor. The stress limit adjustment factor used here, is referred to as a K-factor and is a function of the desired reliability, the confidence requirement and the test sample size. A K-factor equal to three (which implies three standard deviations from the mean value) is assumed here without specifying these inputs. As a comparison, if a reliability of $99 \%$, with a $95 \%$ confidence level (A-basis allowable) is desirable, and the material failure is governed by a normal distribution, about 35 tests are required to obtain a K-factor of three. ${ }^{6}$

If we assume that the uncertainty in the load and the failure stress are normally distributed, the design load can be obtained from:

$$
\tilde{L}=S F\left(\mu_{L}+3 s_{L}\right)
$$

where $\mu_{L}+3 s_{L}$ is defined as the limit load. Launch vehicles typically makes use of a limit load of three standard deviation loads from trajectory simulations with dispersion. Aircraft have a larger empirical database that they can use. For the purposes of this paper, a limit load of three standard deviation loads from the mean load was used, similar to the approach used for launch vehicles. The allowable failure stress could be obtained from:

$$
\tilde{\sigma}_{f a i l}=\mu_{\sigma_{f a i l}}-3 s_{\sigma_{f a i l}}
$$

In Eq. 2 the safety factor $S F$ is applied to the limit load, which in the present work is defined as three standard deviations $s_{L}$ above the mean value $\mu_{L}$ of the service load $L$. In Eq. 3 an allowable failure stress, similar to the A- or B-basis approach, is defined. The allowable failure stress is defined as three standard deviations $s_{\sigma_{f a i l}}$ below the mean value $\mu_{\sigma_{\text {fail }}}$ of the failure stress $\sigma_{\text {fail }}$. Note that the deterministic design process typically does not directly account for uncertainty in problem parameters other than the applied load and stress allowable.

In contrast, RBDO does not make use of safety factors, but instead directly accounts for the uncertainty in all problem parameters to obtain a probability of failure. The equivalent 
RBDO formulation for Eq. 1 would be:

$$
\begin{aligned}
\text { Minimize: } & f(\boldsymbol{x}) \\
\text { Such That: } & P(F) \leq P_{\text {req }} \\
& x_{i}^{\text {lower }} \leq x_{i} \leq x_{i}^{\text {upper }} \quad i=1, n
\end{aligned}
$$

where the probability of failure $P(F)$ is constrained to be less than the allowed probability of failure $P_{\text {req }}$ specified by the designer.

In the present work, the deterministic design approach is simulated by first solving each example problem using the deterministic approach outlined in Eq. 1. For the deterministic design, the design load and failure stress allowable are obtained from Eqs. 2 and 3 respectively.

The deterministic design is followed by a reliability analysis, where all random variables are assumed to be independent and normally distributed with known distributions. Although independent, normally distributed random variables are assumed in the present work, dependent and non-normal variables could also be considered. These variables could be transformed into independent normal variables, using for example the Rosenblatt $^{7}$ transformation. The results of the deterministic design are assumed to be the mean quantities in the reliability evaluation of the deterministic design. The resulting probability of failure is then used as the probability of failure constraint $P_{\text {req }}$ in Eq. 4. The idea is thus to obtain a RBDO design with the same reliability as the corresponding deterministic design. Finally, the proof test data will be accounted for in an attempt to realize further weight savings.

\section{Influence of Proof Testing}

When calculating the probability of failure for a structural component, one is interested in the probability that the applied load is larger than the strength of the structure. The probability of failure is denoted by $P(F)$. Schematically, a qualitative representation of the failure region can be represented as shown in Fig. 1, with the failure region indicated by the shaded area.

When performing a successful proof test, the strength of the component that was proof tested is known to be larger than the load value at which the proof test was conducted. If one assumes that the component either fails the proof test or remains in pristine condition after the proof test, the proof test in effect cuts off the tail of the strength distribution as illustrated in Fig. 2. The assumption that a component that passes the proof test is not damaged by the proof test will be used throughout this paper. This is a critical assumption 


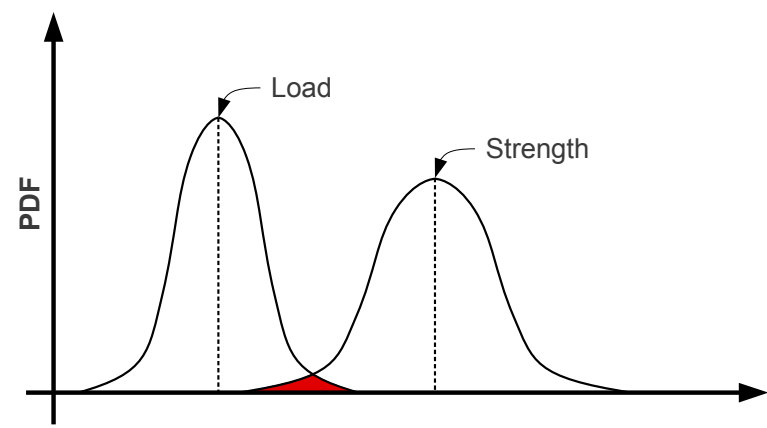

Figure 1. Probability density distributions for stress due to applied load, and structural strength (Region contributing to probability of failure indicated by shading)

that should be noted. Accounting for the effect of damage as a result of the proof test will be the subject of future research.

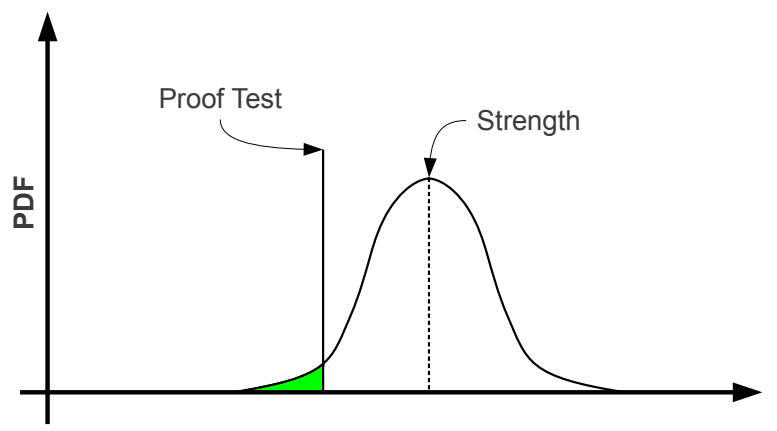

Figure 2. Influence of proof test on strength distribution (Region indicating the probability of failing the proof test $1-P(A)$ is shaded)

The effect of the proof test on calculating the probability of failure after the proof test is performed, is shown in Fig. 3. Note that the failure region is now much smaller than that shown in Fig. 1.

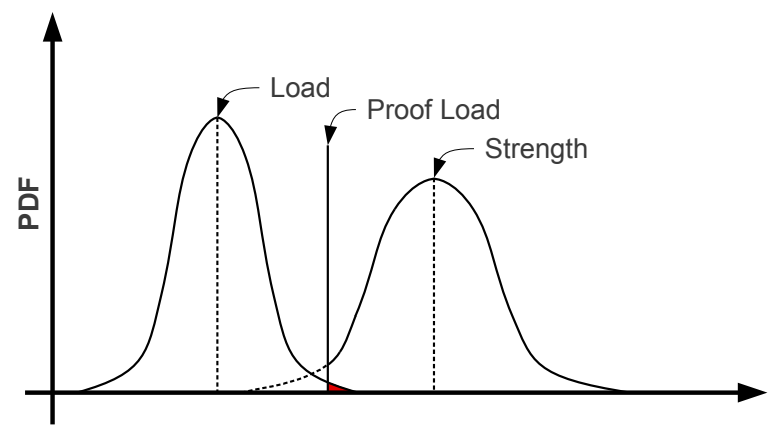

Figure 3. Probability density distributions for stress due to applied load, and structural strength including proof test effect (Region contributing to probability of failure indicated by shading. Note that Fig. 1 denotes $P(F)$, while Fig. 3 denotes the conditional probability $P(F \mid A)$ )

The probability of failure shown in Fig. 3, can be expressed as a conditional probability 
of failure, denoted by $P(F \mid A)$. This conditional probability of failure can be stated as the probability of failure (condition $F$ ), provided that the proof test was successful (condition A). The proof test is successful if the component does not fail and is not damaged when the test is performed. When considering the proof test results in the design process, the probability of failure of the structure is obtained from the conditional probability of failure $P(F \mid A)$ rather than from the probability of failure $P(F)$ as was the case when no proof test data was considered.

The deterministic design approach thus reduces the probability of failure by making use of proof tests. However, this reduction in the probability of failure is not quantified and is thus not directly included in the design process. In the present work, this reduction in the probability of failure will be quantified by calculating the $P(F \mid A)$ value and by using this value in the design process to realize additional weight savings. The RBDO formulation when including the proof load results, can then be written as:

$$
\begin{aligned}
\text { Minimize: } & f(\boldsymbol{x}) \\
\text { Such That: } & P(F \mid A) \leq P_{\text {req }} \\
& 1-P(A) \leq P_{\text {proof }} \\
& x_{i}^{\text {lower }} \leq x_{i} \leq x_{i}^{\text {upper }} \quad i=1, n \\
& p_{j}^{\text {lower }} \leq p_{j} \leq p_{j}^{\text {upper }} \quad j=1, m
\end{aligned}
$$

where $1-P(A)$ is the probability of failing the proof test, $P_{\text {proof }}$ is the maximum acceptable probability of failing the proof test and $p_{j}$ are the variables that describe the magnitude of the proof load. When compared to Eq. 4, this new RBDO formulation has an additional constraint that controls the probability of failing the proof test and includes extra design variables $p_{j}$ that describe the magnitude of the proof test loads. The designer thus has direct control over the probability of failing the proof test and the magnitude of the proof test loads are designed simultaneously with the structural component.

\section{Calculating the Probability of Failure}

Since the calculation of the probability of failure is a costly exercise that requires multiple function evaluations, it is no surprise that the literature provides many methods for efficient calculation of the probability of failure. These methods typically have to manage a trade-off between numerical efficiency and accuracy. Efficient evaluation of the probability of failure is especially important in an optimization framework, since the computational cost is compounded by the fact that a two-level optimization process is encountered in RBDO. At the outer level is the structural optimization problem and at the inner level the 
calculation of the probability of failure.

The focus of the current study is not the efficient calculation of the probability of failure, but rather the effect of including the proof test data in the RBDO process. As a result, it was decided to use a well established and popular method for calculating the probability of failure. The method selected here is the first order reliability method (FORM) ${ }^{8}$ It should be noted that although the FORM approach is widely used, it may not converge to the most probably point of failure (MPP) for cases with complex limit state functions. For problems with complex limit state functions, system reliability techniques based on fault or event trees and numerical techniques tailored to the specific problem at hand may be required.

The FORM method operates in the standard normal space where all random variables are assumed to be independent normal variables that are scaled to have a mean value of 0 and a standard deviation of 1 . A normal distribution with a mean of 0 and a standard deviation of 1 is denoted by $N(0,1)$. The FORM method estimates the probability of failure using a linear approximation of the limit state function $G(X)$ at the most probable point of failure. Where the upper case $X$ is used to denote a random variable. In the present work the limit state function is defined in terms of the stress constraint as:

$$
G(X)=\sigma_{f a i l}(X)-\sigma_{\max }(X)
$$

The limit state is defined as $G(X)=0$ and provides the interface between the failure $G(X)<0$ and safe $G(X)>0$ regions of the design space. Note that there is a different convention between the constraint definition for the deterministic design process and the limit state function used in RBDO. In Eq. 1 the stress constraint is defined to be violated when it has a positive value. In Eq. 6 the limit state function is defined to be violated when it has a negative value. This difference was applied here for consistency with the existing literature.

The FORM algorithm used here is a straight-forward implementation of the algorithm outlined in Haldar and Mahadevan. ${ }^{9}$ This algorithm makes use of a Newton-Raphson type iteration scheme to find the MPP.

When using the FORM approach, the linearized limit state function is a linear combination of independent, normal, random variables and as a result is also a normally distributed random variable. The estimated probability of failure $P(F)$ obtained from the FORM method can then be calculated numerically using Eq. 7:

$$
P(F)=P\left(\bar{G}^{\prime} \geq \beta\right)=\int_{\beta}^{\infty} \frac{1}{\sqrt{2 \pi}} \exp \left[-\frac{1}{2}\left(\bar{g}^{\prime}\right)^{2}\right] d \bar{g}^{\prime}
$$

where $\beta$ indicates the shortest distance from the origin of the standard normal space to the 
limit state function and is obtained from the FORM method. The upper case $\bar{G}^{\prime}$ denotes the random variable that describes the linearized limit state function in the standard space and the lower case $\bar{g}^{\prime}$ denotes an instance of that random variable. The integral on the right hand side of Eq. 7 is simply the probability density function of a normally distributed random variable in the standard space.

To evaluate the conditional probability of failure $P(F \mid A)$, it is necessary to use the multiplication rule (e.g., Haldar and Mahadevan ${ }^{9}$ ), which states that the joint probability $P(F \cap A)$ of $F$ and $A$ is equal to:

$$
P(F \cap A)=P(F \mid A) P(A)
$$

where $P(A)$ can be obtained directly from the FORM method as outlined above. $P(F \cap A)$ can be obtained from:

$$
\begin{gathered}
P(F \cap A)=P\left(\left(\bar{G}_{1}^{\prime} \geq \beta_{1}\right) \cap\left(\bar{G}_{2}^{\prime} \geq \beta_{2}\right)\right)= \\
\int_{\beta_{2}}^{\infty} \int_{\beta_{1}}^{\infty} \frac{|\boldsymbol{R}|^{1 / 2}}{2 \pi} \exp \left[-\frac{1}{2} \overline{\boldsymbol{g}}^{\prime T} \boldsymbol{R}^{-1} \overline{\boldsymbol{g}}^{\prime}\right] d \bar{g}_{1}^{\prime} d \bar{g}_{2}^{\prime}
\end{gathered}
$$

which is simply the integral of the bi-variate normal probability density function in the standard space. Within the current framework, $F$ and $A$ are guaranteed to be normal variables, due to the fact that they are obtained from linearized limit state functions. To evaluate Eq. 9, it is first necessary to perform two FORM analyses to obtain $P(F)$ and $P(A)$ respectively. These two FORM analyses also provide the values for $\beta_{1}$ and $\beta_{2}$ as well as the information required to construct the correlation matrix, $R$. The correlation matrix is a symmetric matrix with all diagonal elements equal to one. For the bi-variate case of Eq. 9 , this is a $2 \times 2$ matrix and only a single entry $r_{12}$ is unknown. The $r_{12}$ value can be obtained from the dot product of the gradients of the linearized limit state functions (e.g., Pandey ${ }^{10}$ ). As was the case for Eq. 7, no analytical solution exists for this integral. However, several very efficient numerical integration schemes are available (e.g., the algorithms provided by Donnelly, ${ }^{11}$ Drezner and Wesolowsky ${ }^{12}$ and Cox and Wermuth ${ }^{13}$ ).

\section{Calculating the System Probability of Failure}

So far the conditional probability of failure has been considered for the special case where only a single service load failure $F$ and a single proof load $A$ was considered. In general, however, multiple service load failures $\boldsymbol{F}$ and multiple proof loads $A$ are possible. When considering more then one failure mode, one needs to consider a system reliability approach to obtain the probability of failure for the component. There are many system re- 
liability approaches available, but the two that are most often encountered are a series and a parallel approach (e.g., Haldar and Mahadevan ${ }^{9}$ ). Serial system failure occurs when any of the failure modes are violated and the resulting probability of system failure represents a union of all the failure events as illustrated in Eq. 10:

$$
P_{s}(\boldsymbol{F})=P\left(F_{1} \cup F_{2} \cup \ldots \cup F_{m f}\right)
$$

Parallel system failure occurs when all of the failure modes are violated and the resulting probability of system failure represents an intersection of all the failure modes as illustrated in Eq. 11:

$$
P_{p}(\boldsymbol{F})=P\left(F_{1} \cap F_{2} \cap \ldots \cap F_{m f}\right)
$$

The parallel system failure $P_{p}(\boldsymbol{F})$ can easily be evaluated from:

$$
P_{p}(\boldsymbol{F})=\int_{\beta_{m f}}^{\infty} \ldots \int_{\beta_{1}}^{\infty} \frac{|\boldsymbol{R}|^{1 / 2}}{(2 \pi)^{m f / 2}} \exp \left[-\frac{1}{2} \overline{\boldsymbol{g}}^{T} \boldsymbol{R}^{-1} \overline{\boldsymbol{g}}^{\prime}\right] d \bar{g}_{1}^{\prime} \ldots d \bar{g}_{m f}^{\prime}
$$

which is the same integral as shown in Eq. 9, but now generalized for $m f$ failure modes, with the correlation matrix $\boldsymbol{R}$ being a $m f \times m f$ matrix. Unlike the uni- and bi-variate integrals of Eqs. 7 and 9, the multi-variate integral of Eq. 12 can be more difficult to evaluate, especially for larger values of $m f$. Typically, this multi-variate integral is evaluated using one of three approaches (e.g. Gassmann ${ }^{14}$ ):

1. Bounding the answer (e.g. Ditlevsen, ${ }^{15}$ Ramachandran ${ }^{16}$ and Ditlevsen ${ }^{17}$ )

2. Approximating the answer (e.g. Hohenbichler and Rackwitz, ${ }^{18}$ Tang and Melchers ${ }^{19}$ and Pandey ${ }^{10}$ )

3. Performing numerical integration (e.g. Drezner ${ }^{20}$ and $\mathrm{Genz}^{21}$ )

Numerical integration can be costly and is typically limited to between 100 and 500 failure modes. In the present work, numerical integration is performed using the algorithm developed by Genz. ${ }^{21}$ The Genz algorithm ${ }^{21}$ presents an efficient numerical integration scheme for solving Eq. 12 for larger values of $m f$, up to 500. Numerical integration is a reasonable approach here, since the number of failure modes considered is in the order of 10. For larger problems, the bounding or approximation approaches should be considered instead.

The series system failure $P_{s}(\boldsymbol{F})$ can not be obtained directly from Eq. 12, since the definition is based in the union of the failure modes instead of the intersection as was the case for the parallel system failure $P_{p}(\boldsymbol{F})$. However, two approaches are available that can be used to convert $P_{s}(\boldsymbol{F})$ into a form that can be evaluated by Eq. 12 . The first approach is 
to make use of the complement of the failure modes $\overline{\boldsymbol{F}}$ to obtain:

$$
P_{S}(\boldsymbol{F})=1-P_{S}(\overline{\boldsymbol{F}})
$$

In addition, De Morgan's rule (e.g., Haldar and Mahadevan ${ }^{9}$ ) can be used to expand the complement as follows:

$$
P_{s}(\overline{\boldsymbol{F}})=P\left(\overline{F_{1} \cup F_{2} \cup \ldots \cup F_{m f}}\right)=P\left(\bar{F}_{1} \cap \bar{F}_{2} \cap \ldots \cap \bar{F}_{m f}\right)
$$

which is in a form that can be evaluated by Eq. 12 .

The second approach is to make use of the definition of the probability of the union of two or more events. For example, for two failure modes this would provide (e.g., Haldar and Mahadevan ${ }^{9}$ ):

$$
P_{s}(F)=P\left(F_{1} \cup F_{2}\right)=P\left(F_{1}\right)+P\left(F_{2}\right)-P\left(F_{1} \cap F_{2}\right)
$$

For this example the first two terms on the right hand side can be evaluated using the uni-variate integral of Eq. 7, while the third term can be evaluated using the bi-variate integral of Eq. 9. In general, when considering more than two failure modes, the terms on the right hand side will include more than two failure events and the multi-variate integral of Eq. 12 can be used.

The advantage of using the first approach is that only a single multi-variate integral is required. The disadvantage is that two numbers that are very close to each other are subtracted, with a potential loss in significant digits. The advantage of the second approach is that the problem with subtracting two numbers that are close to each other is avoided. The disadvantage is that the number of terms on the right hand side grows exponentially as the number of failure modes increases. For example, for three failure modes the number of terms is equal to seven. However, the first order terms $\left(P\left(F_{1}\right)\right.$ and $P\left(F_{2}\right)$ in the above example) are the most important and an upper bound to $P_{s}(\boldsymbol{F})$ can be obtained by considering only these terms. The advantage then would be that the number of terms grow linearly with the number of failure modes and that one only has to perform a number of numerically efficient uni-variate integrals rather than one numerically expensive multi-variate integral.

When the conditional probability of failure $P(\boldsymbol{F} \mid \boldsymbol{A})$ that occurs when accounting for multiple failure modes and multiple proof tests is considered, it is also necessary to deal with both the series and parallel system reliability definitions. For the parallel system, this 
results in:

$$
P_{p}(\boldsymbol{F} \mid \boldsymbol{A})=\frac{P(\boldsymbol{F} \cap \boldsymbol{A})}{P(A)}=\frac{P\left(\left(F_{1} \cap F_{2} \cap \ldots \cap F_{m f}\right) \cap\left(A_{1} \cap A_{2} \cap \ldots \cap A_{m p}\right)\right)}{P\left(A_{1} \cap A_{2} \cap \ldots \cap A_{m p}\right)}
$$

where $m p$ represents the number of proof test failure modes. Equation 16 can be evaluated using Eq. 12. Note that the conditional probability of failure requires the evaluation of two separate multi-variate integrals, one for the numerator and one for the denominator. The numerator has $m f+m p$ terms, while the denominator has $m p$ terms.

For the series case the conditional probability results in:

$$
P_{s}(\boldsymbol{F} \mid \boldsymbol{A})=\frac{P\left(\left(F_{1} \cup F_{2} \cup \ldots \cup F_{m f}\right) \cap\left(A_{1} \cap A_{2} \cap \ldots \cap A_{m p}\right)\right)}{P\left(A_{1} \cap A_{2} \cap \ldots \cap A_{m p}\right)}
$$

As was the case for $P_{s}(\boldsymbol{F})$, there are two strategies that can be used to convert Eq. 17 into a form where the multi-variate integral of Eq. 12 is applicable. For the first approach, the complement and De Morgan's rule is used similar to Eqs. 13 and 14 to obtain:

$$
P_{s}(\boldsymbol{F} \mid A)=1-P(\overline{\boldsymbol{F}} \mid \boldsymbol{A})=1-\frac{P\left(\left(\bar{F}_{1} \cap \bar{F}_{2} \cap \ldots \cap \bar{F}_{m f}\right) \cap A\right)}{P(A)}
$$

For the second approach, the definition for the probability of the union of events is used similar to Eq. 15. For the special case where two failure modes are considered, this results in:

$$
P_{s}(\boldsymbol{F} \mid A)=\frac{P\left(F_{1} \cap A\right)}{P(A)}+\frac{P\left(F_{2} \cap A\right)}{P(A)}-\frac{P\left(\left(F_{1} \cap F_{2}\right) \cap A\right)}{P(A)}
$$

In both cases the multi-variate integral of Eq 12 can be used to evaluate the numerator and denominator of the right hand side terms. As was the case when evaluating $P_{s}(\boldsymbol{F})$, the same advantages and disadvantages exist for each approach. However, numerical experimentation has shown that the first approach is problematic. The numerical integration of the numerator in Eq. 18 was found to be difficult and the Genz algorithm has trouble converging. Although the second approach could have a large number of terms on the right hand side, it has the advantage that the Genz algorithm seems to converge very easily for all of these terms. As a result, the second approach is used throughout this paper. Furthermore, it is shown that for the example problems considered here, that an insignificant loss of accuracy is incurred by creating an upper bound for $P_{s}(\boldsymbol{F} \mid A)$ using:

$$
P_{S}(\boldsymbol{F} \mid A)=\min \left(1.0, \sum_{i=1}^{m f} P\left(F_{i} \mid \boldsymbol{A}\right)\right)
$$




\section{Numerical Examples}

Two analytic example problems are considered to illustrate the proposed method. The first is a simple cantilevered beam that has a single load condition with a single failure mode and thus illustrates the use of $P(F \mid A)$. The second is a stepped cantilevered beam, that also has a single load condition but with multiple failure regions for both the service and the proof loads. For the second example, a series system reliability approach is followed and thus the use of $P_{s}(\boldsymbol{F} \mid \boldsymbol{A})$ is illustrated. In both cases, a single, uniform material is assumed and the volume instead of the weight is used as objective function. When using a single, uniform material, minimizing the volume is equivalent to minimizing the weight.

In both cases, a deterministic design is performed first, using the limit load and safety factor approach of Eq. 2 and the allowable failure stress of Eq. 3. The probability of failure for these deterministic designs are then determined using the FORM approach and is used as the required probability of failure $P_{\text {req }}$ for the RBDO design that follows.

\section{A. Example 1: Cantilevered Beam Problem}

The first example problem is a simple linear, homogeneous and isotropic cantilevered beam with a uniform cross-section as illustrated in Fig. 4 . The beam has length $l$, width $b$, height $h$ and is subject to a service load $L$ at the tip.

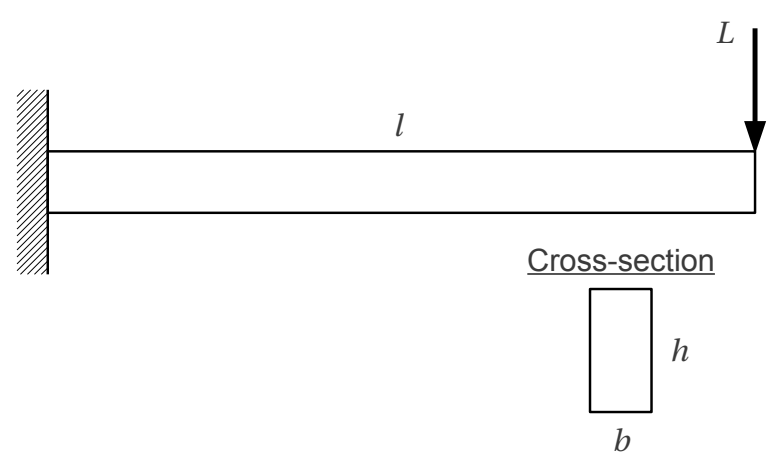

Figure 4. Cantilevered beam

It is assumed that the beam has random variables that are both independent and normal with the distribution data outlined in Table 1. Note that all variables have a fixed standard deviation. Also, the mean values of the width and height are not yet known and will be determined from the optimization process. The standard deviation of the geometric variables represent machining tolerances and although a uniform distribution may have been more appropriate, a normal distribution is used in the present work. 
Table 1. Random variable definition

\begin{tabular}{cc}
\hline Parameter & Distribution \\
\hline \hline Load $(L)$ & $N(1000,100) \mathrm{N}$ \\
Length $(l)$ & $N(500.0,2.0) \mathrm{mm}$ \\
Width $(b)$ & $N(?, 1.0) \mathrm{mm}$ \\
Height $(h)$ & $N(?, 1.0) \mathrm{mm}$ \\
Failure Stress $\left(\sigma_{\text {fail }}\right)$ & $N(350,25) \mathrm{MPa}$ \\
\hline
\end{tabular}

The first step is to perform a deterministic design of the beam as follows:

$$
\begin{aligned}
\text { Minimize: } & \text { Volume } \\
\text { Such That: } & \frac{h}{b} \leq 2 \\
& \sigma_{\max } \leq \tilde{\sigma}_{\text {fail }} \\
& b, h \geq 0
\end{aligned}
$$

where the first constraint is a simple geometric constraint to ensure stability and:

$$
\begin{aligned}
& \sigma_{\max }=\frac{6 \tilde{L} l}{b h^{2}} \\
& \tilde{L}=1.4\left(\mu_{L}+3 s_{L}\right) \\
& \tilde{\sigma}_{\text {fail }}=\mu_{\sigma_{f a i l}}-3 s_{\sigma_{\text {fail }}}
\end{aligned}
$$

The deterministic optimization was performed using a safety factor of 1.4 for the load, the mean values of the quantities in Table 1 and the DOT $^{22}$ optimizer. The results are summarized in Table 2. Both constraints are active at the optimum.

Table 2. Deterministic optimization results

\begin{tabular}{cc}
\hline Parameter & Value \\
\hline \hline Width $(b)$ & $17.1 \mathrm{~mm}$ \\
Height $(h)$ & $34.1 \mathrm{~mm}$ \\
\hline
\end{tabular}

To determine the probability of failure $P(F)$ for the deterministic design outlined in Table 2, a FORM analysis was performed using the distribution data of Table 1 . The resulting probability of failure was found to be $P(F)=7.12 \times 10^{-9}$. Using this probability of failure as $P_{\text {req }}$ in Eq. 4 resulted in a RBDO design that was the same as the deterministic design. For this simple example problem, there is thus no advantage moving from the deterministic to an equivalent RBDO design.

Before continuing to the RBDO design that includes the proof test data, a numerical 
experiment was performed to determine the accuracy of the assumptions outlined in this paper for evaluating $P(F \mid A)$. For this experiment, a deterministic optimization was performed, but using $\mu_{\sigma_{f a i l}}$ instead of $\tilde{\sigma}_{\text {fail }}$ to obtain a design with a probability of failure $P(F)=3.88 \times 10^{-5}$ instead of $7.12 \times 10^{-9}$. This was done to obtain a probability of failure value that could be validated with a reasonable number of Monte Carlo simulations. The accuracy of the Monte Carlo simulations was evaluated by calculating the coefficient of variation (COV) using Eq. 23, obtained from Haldar and Mahadevan. ${ }^{9}$

$$
\operatorname{COV}\left(P_{f}\right)=\delta_{P_{f}}=\frac{\sqrt{\frac{\left(1-P_{f}\right) P_{f}}{M}}}{P_{f}}
$$

In Eq. $23 P_{f}$ is the probability of failure as estimated by the Monte Carlo simulation and $M$ is the number of Monte Carlo analyses that was performed. The Monte Carlo results are compared to the probability of failure $P(F)$, the probability of failing the proof test $1-P(A)$ and the conditional probability of failure $P(F \mid A)$ computed using the proposed FORM based approach. For this experiment, five Monte Carlo simulations were considered. Due to the different magnitudes of the estimated probability of failure values obtained, different numbers of analyses were considered for each of the Monte Carlo simulations. For the first simulation $5 \times 10^{6}$ analyses was considered, for the $2^{\text {nd }}, 3^{\text {rd }}$ and $4^{\text {th }}$ simulations $25 \times 10^{6}$ analyses and for the $5^{\text {th }}$ simulation $75 \times 10^{6}$ analyses. The average COV values for the five simulations were 0.04 for $P(F), 0.10$ for $1-P(A)$ and 0.01 for $P(F \mid A)$. The results are summarized in Fig. 5 below.

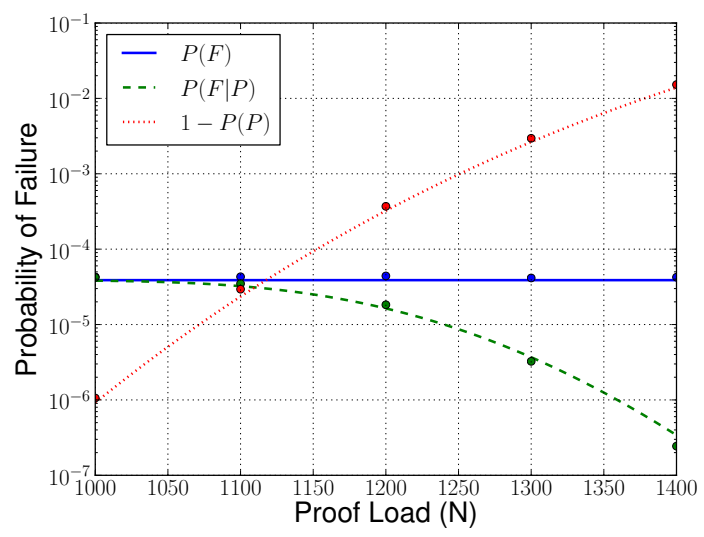

Figure 5. FORM analysis validation using Monte Carlo simulations (solid lines from FORM analysis, dots from Monte Carlo simulations)

In Fig. 5, the y-axis provides the probability of failure and has a logarithmic scale. The $\mathrm{x}$-axis specifies the proof load value. For this example, the proof load is defined as a point load that is applied at the same location and in the same direction as the service load and 
varies from the mean value of the service load $(1000 \mathrm{~N})$ to 1.4 times the mean value of the service load (1400 N). For reference, the limit load has a value of 1300 N. From Fig. 5 it is clear that the $P(F)$ value is not influenced by the proof load. However, as the proof load is increased, the probability of failing the proof load $1-P(A)$ is also increased, while the conditional probability of failure $P(F \mid A)$ is reduced. Considering the COV values for the Monte Carlo simulations, all three cases show good correlation between the FORM based and the Monte Carlo results. Clearly the Monte Carlo simulations validate the overall trend obtained from the FORM based approach.

To account for the proof test data, the following RBDO design problem was defined:

$$
\begin{array}{rlr}
\text { Minimize: } & \text { Volume } \\
\text { Such That: } & \frac{h}{b} \leq 2 \\
& P(F \mid A)<7.12 \times 10^{-9} \quad \\
& 1-P(A)<P_{\text {proof }} & \text { (Conditional POF) } \\
& b, h, p \geq 0
\end{array}
$$

where the required probability of failure was obtained from the deterministic design and the required probability of failing the proof test $P_{\text {proof }}$ can be set by the designer. In this work, the value of $P_{\text {proof }}$ will be varied to obtain a trade-off graph. Note that the magnitude of the proof load $p$ is also included as a design variable. The results of the trade-off study when varying the value of $P_{\text {proof }}$ in Eq. 24 is summarized graphically in Fig. 6.

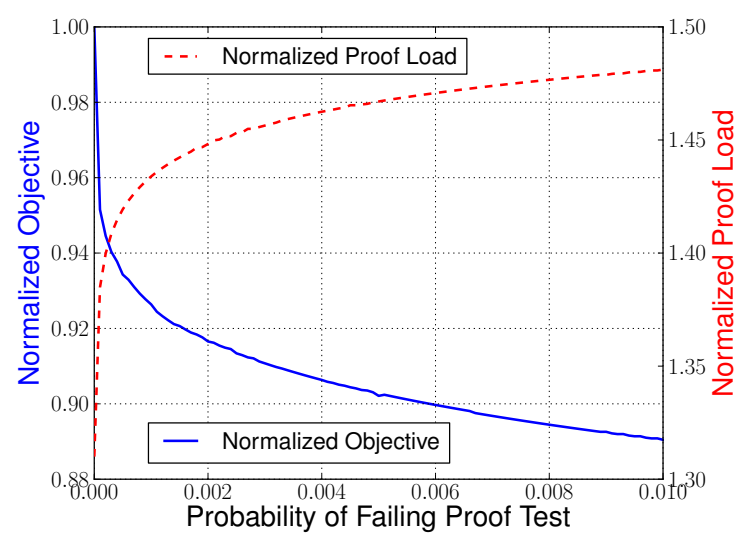

Figure 6. Trade-off study for different values of $P_{\text {proof }}$ (with optimized proof load)

In Fig. 6 the objective function is normalized with respect to the deterministic optimum, while the proof load is normalized with respect to mean value of the service load. As a reference, the limit load would have a normalized value of 1.3 and a proof test load of 1.2 
times the limit load would have a normalized value of 1.56 .

From Fig. 6 it is clear that by including the proof test data as part of the RBDO design process, that it is possible to realize significant weight savings while maintaining the same high reliability obtained from the original deterministic design. Also, the normalized objective function is reduced very quickly for relatively small probabilities of failing the proof test, with diminishing returns for higher probabilities of failing the proof test. Finally, when designing both the structure and the proof test at the same time, the optimizer prefers to use lower values for the proof test than one would typically expect. The standard proof test value would be 1.2 times the limit load. ${ }^{23}$ In Fig. 6 all tests are performed below this threshold. Armed with a trade-off graph like Fig. 6, the designer can make informed decisions as to what weight savings can be realized for an acceptable probability of failing the proof test.

The results of Fig. 6 should also be compared to the current way of proof testing, where a fixed proof load is used. The trade-off study for a fixed proof load equal to 1.2 times the limit load is shown in Fig. 7. From Fig. 7 it is clear that even with the current approach

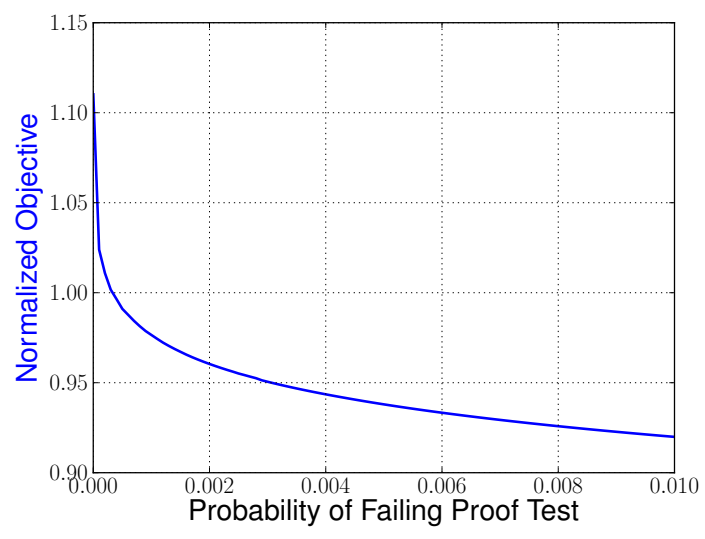

Figure 7. Normalized objective function for different values of $P_{\text {proof }}$ using a fixed proof load

of using a fixed proof test load, significant weight saving is possible. Also, Fig. 7 has great value in helping the designer manage the risk associated with performing the proof test. However, when compared to Fig. 6, it is clear that additional weight savings is possible when also considering the proof test load as a design variable.

\section{B. Example 2: Stepped Cantilevered Beam Problem}

The second example problem is a stepped cantilevered beam with three segments. The beam is also linear, homogeneous and isotropic and each of the three segments are assumed to have a uniform cross-section. The height of each segment $h_{i}$ is different, while the beam has a constant width $b$ throughout. The length of the beam is $l=0.5 \mathrm{~m}$ and 
is not considered as a random variable. The beam is subject to a uniformly distributed service load $q$ and a proof load that consists of three components $L_{1}, L_{2}$ and $L_{3}$. These three load components represent three actuators that are used to simulate the service load during the proof test. As a result, the relative magnitude of the three load components are fixed as shown in Fig. 8. The relative magnitude between the three load components were obtained from a lumped equivalent work approach (e.g., Cook, Malkus and Plesha ${ }^{24}$ ) similar to what one would use when modeling the beam with three finite elements. The actual proof load is obtained by multiplying a single scale factor with the relative magnitude of the load components to obtain the values for $L_{i}$.

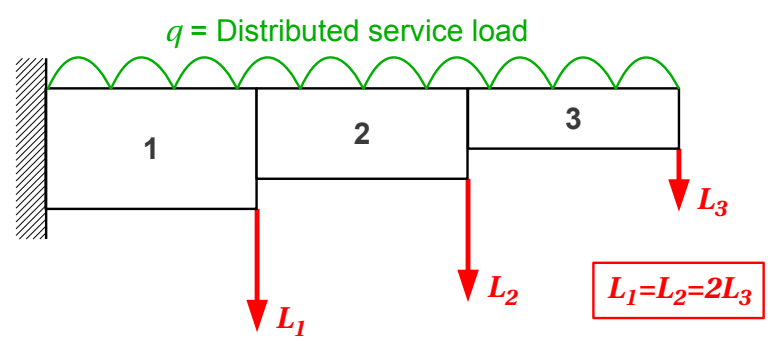

Figure 8. Stepped cantilevered beam

As for the first example, the random variables are assumed to be normally distributed and independent with the distribution data summarized in Table 3. The mean values of the height and width variables are not yet known and will be determined from the optimization process.

Table 3. Random variable definition

\begin{tabular}{cc}
\hline Parameter & Distribution \\
\hline \hline Load $(q)$ & $N(7500,750) \mathrm{N} / \mathrm{m}$ \\
Width $(b)$ & $N(?, 1.0) \mathrm{mm}$ \\
Height $\left(h_{1}\right)$ & $N(?, 1.0) \mathrm{mm}$ \\
Height $\left(h_{2}\right)$ & $N(?, 1.0) \mathrm{mm}$ \\
Height $\left(h_{3}\right)$ & $N(?, 1.0) \mathrm{mm}$ \\
Failure Stress $\left(\sigma_{\text {fail }}\right)$ & $N(350,25) \mathrm{MPa}$ \\
\hline
\end{tabular}

The deterministic optimization is performed first, using the following formulation:

Minimize: Volume

$$
\begin{aligned}
\text { Such That: } & \frac{h_{i}}{b} \leq 2 \quad \text { where } i=1,2,3 \\
& \sigma_{\max _{i}} \leq \tilde{\sigma}_{\text {fail }} \\
& b, h_{i} \geq 0
\end{aligned}
$$


where the first constraint equation represents three geometric constraints to ensure stability and:

$$
\begin{aligned}
& \sigma_{\max _{i}}=\frac{3 \tilde{q}}{b h_{i}^{2}}\left(\frac{(4-i) l}{3}\right)^{2} \\
& \tilde{q}=1.4\left(\mu_{q}+3 s_{q}\right) \\
& \tilde{\sigma}_{\text {fail }}=\mu_{\sigma_{\text {fail }}}-3 s_{\sigma_{\text {fail }}}
\end{aligned}
$$

As was the case for the first example, a safety factor of 1.4 is used for the load.

The DOT results for this deterministic optimization are summarized in Table 4. The optimum design is fully stressed with the failure stress constraint active for each of the three segments.

Table 4. Deterministic optimization results

\begin{tabular}{cc}
\hline Parameter & Value \\
\hline \hline Width $(b)$ & $21.0 \mathrm{~mm}$ \\
Segment 1 Height $\left(h_{1}\right)$ & $42.1 \mathrm{~mm}$ \\
Segment 2 Height $\left(h_{2}\right)$ & $28.0 \mathrm{~mm}$ \\
Segment 3 Height $\left(h_{3}\right)$ & $14.0 \mathrm{~mm}$ \\
\hline
\end{tabular}

Next a FORM analysis was performed to determine the probability of failure for the deterministic design. A series system reliability approach and the distribution data of Table 3 were used. The resulting probability of failure was found to be $P_{s}(F)=2.56 \times 10^{-5}$. With the probability of failure known for the deterministic design, an equivalent RBDO design could be performed using the formulation outlined in Eq. 4. Unlike the first example, the equivalent RBDO for the second example provided a design that differed significantly from the deterministic design. The RBDO design is summarized in Table 5. Table 5 also includes the results from a second RBDO run, where the value of $P_{r e q}$ was changed from $2.56 \times 10^{-5}$ to $1.0 \times 10^{-7}$. The reduced weight associated with the RBDO design is a result of the difference between the two design approaches which is discussed in more detail below.

Table 5 indicates that the equivalent RBDO design with the same $P_{s}(F)$ value as the deterministic design resulted in a $12 \%$ weight savings when compared to the deterministic design. In addition, when reducing the $P_{\text {req }}$ value to $1.0 \times 10^{-7}$, the probability of failure was reduced by roughly two orders of magnitude while maintaining roughly the same weight when compared to the deterministic design. The weight savings that are realized in the RBDO designs are a result of the distribution of the probability of failure values for each of the three segments as outlined in Table 6.

Table 6 clearly illustrates the difference between the two design approaches. The deter- 
Table 5. Reliability based design optimization results

\begin{tabular}{c|cc|c}
\hline Parameter & Deterministic & RBDO $^{1}$ & RBDO $^{2}$ \\
\hline \hline$P_{s}(\boldsymbol{F})$ & $2.56 \times 10^{-5}$ & $2.56 \times 10^{-5}$ & $1.0 \times 10^{-7}$ \\
\hline Width $(b)(\mathrm{mm})$ & 21.0 & 19.3 & 20.3 \\
Height $\left(h_{1}\right)(\mathrm{mm})$ & 42.1 & 38.7 & 40.7 \\
Height $\left(h_{2}\right)(\mathrm{mm})$ & 28.0 & 26.7 & 28.1 \\
Height $\left(h_{3}\right)(\mathrm{mm})$ & 14.0 & 15.0 & 16.1 \\
\hline Volume $\left(\times 10^{-6} \mathrm{~m}^{3}\right)$ & 885.3 & 777.1 & 862.9 \\
\hline \multicolumn{2}{c|}{$R B D O^{1}-R B D O$ design with $P_{\text {req }}=2.56 \times 10^{-5}$} \\
\multicolumn{2}{c}{$R B D O^{2}-R B D O$ design with $P_{\text {req }}=1.0 \times 10^{-7}$} \\
\hline
\end{tabular}

Table 6. Probability of failure values for each segment. Both designs has $P_{S}(F)=2.56 \times 10^{-5}$ (Stress values calculated from the design load in both cases)

\begin{tabular}{c|cc|cc}
\hline & \multicolumn{2}{|c|}{ Deterministic } & \multicolumn{2}{c}{ RBDO } \\
Parameter & Stress (MPa) & $P(F)$ & Stress (MPa) & $P(F)$ \\
\hline \hline Segment 1 & 275 & $7.19 \times 10^{-10}$ & 354 & $1.56 \times 10^{-5}$ \\
Segment 2 & 275 & $9.73 \times 10^{-9}$ & 329 & $7.21 \times 10^{-6}$ \\
Segment 3 & 275 & $2.55 \times 10^{-5}$ & 261 & $4.23 \times 10^{-6}$ \\
\hline
\end{tabular}

ministic design is only concerned with stress and results in a fully stressed design with the same maximum stress value for each segment. In contrast the RBDO design is only concerned with the $P_{s}(F)$ value, which is determined from the $P(F)$ values for each segment. Acar and Haftka ${ }^{25}$ illustrated that for the RBDO design, the ratio of the probability of failure for each segment should be roughly equal to the weight ratio of the segments. The weight and probability of failure ratios of the three segments are summarized in Table 7 below. In both cases, the normalization was performed using the values for segment 3 . Clearly the weight and probability of failure ratios exhibit the same trend as observed by Acar and Haftka. ${ }^{25}$

Table 7. Comparison of weight and probability of failure ratios for the RBDO design (in both cases the values are normalized with that of segment 3 )

\begin{tabular}{lcc}
\hline & $\begin{array}{c}\text { Normalized } \\
\text { Weight }\end{array}$ & $\begin{array}{c}\text { Normalized } \\
P_{s}(\boldsymbol{F})\end{array}$ \\
\hline \hline Segment 1 & 3 & 3.7 \\
Segment 2 & 2 & 1.7 \\
Segment 3 & 1 & 1 \\
\hline
\end{tabular}

Although the $P_{s}(\boldsymbol{F})$ value is the same for both designs, the $P(F)$ values for each segment are significantly different. Even though the maximum stress value is the same for each 
segment in the deterministic design, only segment 3 is critical from a probability of failure point of view, while segments 1 and 2 are over designed. The reason for the different $P(F)$ values is that the standard deviation for the $h_{i}$ values are constant. For segment 3 the mean deterministic design value for $h$ is much smaller than for segment 1 . The fixed standard deviation thus has a much larger influence on the stress value of segment 3 than it does on the stress value of segment 1 , resulting in the higher probability of failure for segment 3. Similarly, due to the smaller variability in the stress value of segment 1 , the RBDO approach allows a higher stress value for this segment, while maintaining a lower stress value in segment 3.

Before continuing to the RBDO design that includes the proof test data, another numerical experiment was conducted to compare the results obtained from the FORM based approach outlined in this paper with a Monte Carlo simulation. For this experiment the RBDO design with $P_{s}(\boldsymbol{F})=2.56 \times 10^{-5}$ was used as a design point, and a proof load that represents the limit load was applied. For the Monte Carlo simulation $10^{8}$ analyses were performed and the results are summarized in Table 8.

Table 8. Comparison of Monte Carlo and FORM results for RBDO design with $P_{S}(F)=2.56 \times 10^{-5}$ and proof load equal to limit load (For the Monte Carlo results, the values in parenthesis indicate the COV values obtained from Eq. 23)

\begin{tabular}{ccc|c}
\hline Parameter & Monte Carlo & FORM & FORM $^{* *}$ \\
\hline \hline$P_{S}(\boldsymbol{F})$ & $2.764 \times 10^{-5}$ & $2.557 \times 10^{-5}$ & $2.701 \times 10^{-5}$ \\
& $(0.019)$ & & \\
$P(A)$ & 0.997827 & 0.998038 & - \\
& $\left(4.667 \times 10^{-6}\right)$ & & \\
$P_{S}(\boldsymbol{F} \mid \boldsymbol{A})$ & $2.916 \times 10^{-6}$ & $3.422 \times 10^{-6}$ & $3.423 \times 10^{-6}$ \\
& $(0.059)$ & & \\
\hline
\end{tabular}

In Table 8, the FORM ${ }^{* *}$ column indicates results obtained from the upper bound approximation for the $P_{s}$ values as outlined in Eq. 20. Table 8 shows excellent correlation between the FORM and FORM** columns, indicating that the upper bound approximation approach for estimating the $P_{S}$ values is valid for the current example problem. In addition, Table 8 shows good correlation between the Monte Carlo and FORM approaches for the $P_{s}(F)$ and $P(A)$ values, with a slightly larger variation for the $P_{s}(F \mid A)$ value. This larger variation can in part be explained by the smaller numerical value associated with $P_{s}(\boldsymbol{F} \mid \boldsymbol{A})$. The Monte Carlo simulation resulted in 2764 failures in $10^{8}$ simulations used for estimating the $P_{s}(\boldsymbol{F})$ value, compared to only 291 failures in $10^{8}$ simulations used for estimating the $P_{s}(\boldsymbol{F} \mid \boldsymbol{A})$ value. The Monte Carlo estimate of $P_{s}(\boldsymbol{F} \mid \boldsymbol{A})$ is thus less accurate than than of $P_{s}(F)$.

The final step for this example problem was to perform the RBDO design that accounts 
for the proof test data. The following formulation was used:

Minimize: Volume

Such That: $\frac{h_{i}}{b} \leq 2$

$$
\begin{array}{lr}
P(\boldsymbol{F} \mid \boldsymbol{A})<1.0 \times 10^{-7} & \text { (Conditional POF) } \\
1-P(A)<P_{\text {proof }} & \text { (Prob of failing any proof test) } \\
b, h_{i}, p \geq 0 &
\end{array}
$$

The $P_{\text {req }}$ value used for the conditional probability of failure in Eq. 27 was obtained from the second RBDO design outlined in Table 5. The magnitude of the proof load is included as a design variable and the allowable probability of failing the proof test $P_{\text {proof }}$ was varied to generate a trade-off as shown in Fig. 9.

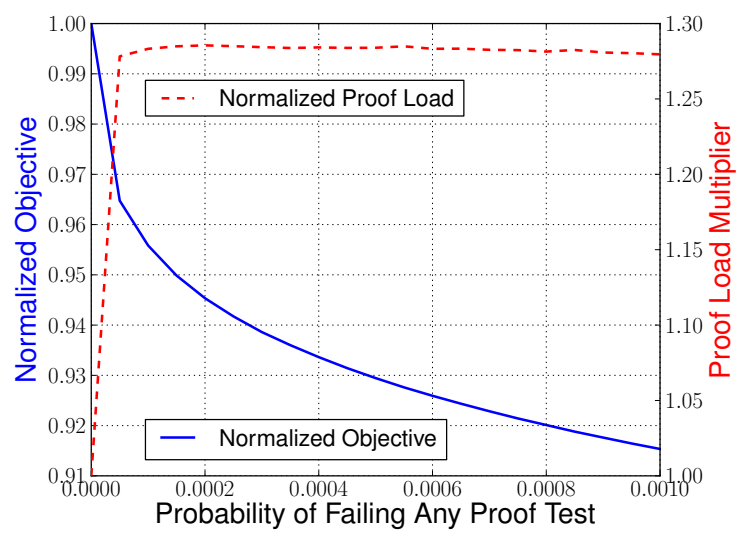

Figure 9. Trade-off study for different values of $P_{\text {proof }}$

In Fig. 9 the $\mathrm{x}$-axis provides the specified probability of failing the proof test. The objective function is normalized with respect to the RBDO design for $P(F)=1.0 \times 10^{-7}$ (the RBDO design already presents a small weight savings over the corresponding deterministic design, but with a significantly higher reliability) and the proof load is normalized with respect to the mean value of the service load. As a reference, a proof test load that corresponds to 1.2 times the limit load would have a normalized value of 1.56.

Figure 9 presents a similar effect on the objective function, as was observed in Fig. 6 with a rapid initial drop-off in weight, followed by diminishing returns for larger proof failure probabilities. Figure 9 indicates roughly an $8 \%$ reduction in weight over the corresponding RBDO design (that does not account for the proof test data) with a probability of 1 in 1000 of failing the proof load. For the proof load, as was the case in Fig. 6, Fig. 9 indicates that the proof tests should be performed at smaller values than what is typically 
used. In this case, the proof load should be just below the limit load, while standard practice is to use a proof load 1.2 times the limit load. It is interesting to note that the traditional use of acceptance testing is to check workmanship. However, the question at which level to perform the acceptance test is generally not known and typically depends on individual experience. Trade-off graphs like those presented in Figs. 6 and 9 may be used as a quantitative tool to help determine and motivate an appropriate level at which to perform the acceptance test. In Fig. 9, the proof load basically remains constant over the full range of $P_{\text {proof }}$ values considered. The optimizer increases the probability of failing the proof test by decreasing the weight rather than by increasing the proof load.

\section{Concluding Remarks}

This paper presents a methodology for including the results of proof/acceptance tests in the RBDO process. The proposed method allows for the simultaneous design of the structural component and the proof test itself and provides the designer with direct control over the probability of failing the proof test. The results indicate that a significant weight saving is possible when including the proof test results in the design process as compared to equivalent deterministic or RBDO designs, while maintaining the same probability of failure as obtained from the deterministic design. Trade-off graphs generated here allows the designer to select an appropriate probability of failing the proof test, based on the project at hand.

Several issues should be further investigated in future work. This includes the assumption that the components remain in pristine condition after the proof test. The use of more realistic distribution data, other than independent, normal distributions, should be explored. For example, other distributions may be more appropriate than the normal distribution used here to represent manufacturing tolerances. Also, analysis techniques other than FORM should be investigated to provide the designer with a wider range of tools for performing the probabilistic analysis. Finally, the method should be applied to more complicated applications that require the use of numerical simulations techniques like finite element analyses.

\section{References}

${ }^{1}$ Grandhi, R. and Wang, L., "Higher-Order Failure Probability Calculation Using Nonlinear Approximations," Computer Methods in Applied Mechanics and Engineering, Vol. 168, No. 1-4, 1999, pp. 185-206.

${ }^{2}$ Acar, E., Haftka, R., Kim, N., and Buchi, D., "Effects of structural tests on aircraft safety," AIAA journal, Vol. 48, No. 10, 2010, pp. 2235-2248. 
${ }^{3}$ Mason, B. H. and Krishnamurthy, T., "Probabilistic Analysis of a Composite Crew Module," Proceedings of the 52nd AIAA/ASME/ASCE/AHS/ASC Structures, Structural Dynamics and Materials Conference, Denver, Colorado, 4-7 April 2011.

${ }^{4}$ Herbert, J. J. and Trilling, L. H., "Development of Factors of Safety for Structural Analysis and Verification of Cryogenic Structures Using Probabilistic Methods," Proceedings of the 47th AIAA/ASME/ASCE/AHS/ASC Structures, Structural Dynamics, and Materials Conference, Newport, Rhode Island, AIAA 2006-1992, May 1-4 2006.

${ }^{5}$ Meyer-Jens, R. J., "Factors of Safety - Historical Development, State of the Art and Future Outlook," AGARD Report No. 661, North Atlantic Treaty Organization - Advisory Group on Aerospace Research and Development, 1977.

${ }^{6}$ Verderaime, V., "Structural Deterministic Safety Factors Selection Criteria and Verification," NASA TP3202, 1992.

${ }^{7}$ Rosenblatt, M., "Remarks on a Multivariate Transformation," Annals of Mathematical Statistics, Vol. 23, No. 3, 1952, pp. 470-472.

${ }^{8}$ Rackwitz, R. and Fiessler, B., "Structural Reliability Under Combined Random Load Sequences," Computers and Structures, Vol. 9, No. 5, 1978, pp. 484-494.

${ }^{9}$ Haldar, A. and Mahadevan, S., Probability, Reliability and Statistical Methods in Engineering Design, John Wiley \& Sons, 2000.

${ }^{10}$ Pandey, M. D., "An Effective Approximation to Evaluate Multinormal Integrals," Structural Safety, Vol. 20, 1998, pp. 51-67.

${ }^{11}$ Donnelly, T., "Algorithm 462: Bivariate Normal Distribution," Communications of the ACM, Vol. 16, No. 10, pp. 638.

${ }^{12}$ Drezner, Z. and Wesolowsky, G., "On the Computation of the Bivariate Normal Integral," Journal of Statistical Computation and Simulation, Vol. 35, No. 1, 1990, pp. 101-107.

${ }^{13}$ Cox, D. and Wermuth, N., "A Simple Approximation for Bivariate and Trivariate Normal Integrals," International Statistical Review, Vol. 59, No. 2, 1991, pp. 263-269.

${ }^{14}$ Gassmann, H., Deák, I., and Szántai, T., "Computing Multivariate Normal Probabilities: A New Look," Journal of Computational and Graphical Statistics, Vol. 11, No. 4, 2002, pp. 920-949.

${ }^{15}$ Ditlevsen, O., "Narrow reliability bounds for structural systems," Mechanics Based Design of Structures and Machines, Vol. 7, No. 4, 1979, pp. 453-472.

${ }^{16}$ Ramachandran, K., "System Bounds: A Critical Study," Civil Engineering and Environmental Systems, Vol. 1, No. 3, 1984, pp. 123-128.

${ }^{17}$ Ditlevsen, O. and Bjerager, P., "Methods of structural systems reliability," Structural Safety, Vol. 3, No. 3-4, 1986, pp. 195-229.

${ }^{18}$ Hohenbichler, M. and Rackwitz, R., "First-Order Concepts in System Reliability," Structural Safety, Vol. 1, No. 3, 1983, pp. 177-188.

${ }^{19}$ Tang, L. K. and Melchers, R. E., "Improved Approximation for Multinormal Integral," Structural Safety, Vol. 4, No. 2, 1986, pp. 81-93.

${ }^{20}$ Drezner, Z., "Computation of the multivariate normal integral," ACM Transactions on Mathematical Software (TOMS), Vol. 18, No. 4, 1992, pp. 470-480.

${ }^{21}$ Genz, A., "Numerical Computation of Multivariate Normal Probabilities," Journal of computational and graphical statistics, Vol. 1, 1992, pp. 141-149. 
${ }^{22}$ DOT. Design Optimization Tools, Version 5.x, Users Manual, Vanderplaats Research and Development, Inc., 1767 S. 8th St., Suite 100, Colorado Springs, CO, January 2001.

23 "Structural Design and Test Factors of Safety for Spaceflight Hardware," NASA Technical Standard, NASA-STD-5001, 1996.

${ }^{24}$ Cook, R. D., Malkus, D. S., and Plesha, M. E., Concepts and Applications of Finite Element Analysis, John Wiley \& Sons, 1989.

${ }^{25}$ Acar, E. and Haftka, R., "Reliability-based aircraft structural design pays, even with limited statistical data," Journal of aircraft, Vol. 44, No. 3, 2007, pp. 812-823. 